\title{
Comparison of Intraoperative Nociception and Postoperative Acute Pain after Traditional or Minimally Invasive Ovariohysterectomy in Dogs
}

\author{
Bruno Watanabe Minto Fabrícia Geovânia Fernandes Filgueira $_{\odot}$ \\ Brenda Mendonça de Alcântara ${ }_{\odot}$ Laís Fernanda Sargi



\begin{abstract}
Background: Many variations of ovariohysterectomy techniques have been described, including the traditional one and minimally invasive procedures. Non-laparoscopic Snook hook technique is an alternative for performing minimally invasive ovariohysterectomy. Few studies have been carried out in order to assess pain in animals submitted to minimally invasive surgeries, especially involving one of the most performed surgical procedures in veterinary practice. The aim of this study was to evaluate surgical duration, intraoperative nociception and acute postoperative pain after traditional ovariohysterectomy or minimally invasive non-laparoscopic technique in dogs using Snook hook. The hypothesis is that non-laparoscopic minimally invasive ovariohysterectomy would be faster and less painful than the conventional technique. Material, Methods \& Results: Thirty dogs were divided into Traditional Group (TG $=15)$ and Minimally Invasive Group $(\mathrm{MIG}=15)$. Heart rate, respiratory rate, systolic blood pressure, body temperature, oxyhemoglobin saturation, end-tidal carbon dioxide concentration (ETCO2) and end-tidal isoflurane concentration were evaluated before the surgery begins (M0), during incision (M1), clamping of the first ovarian pedicle (M2), second ovarian pedicle (M3), uterine cervix (M4), abdominal suture (M5) and at the end of surgery (M6). The modified Glasgow Pain Scale was used for acute postoperative pain assessment and Visual Analogue Scale (VAS) was used to assess the sensitivity of surgical wound. The level of significance established for all statistical analysis was 5\%. Statistical differences were not observed between groups considering total surgical time and postoperative acute pain intensity $(P>0.05)$, in spite of MIG having shorter duration of surgery. There was no statistical difference between groups considering all intraoperative parameters except respiratory rate $(\mathrm{TG}<\mathrm{MIG} ; P<0.05)$ and ETCO2 $(\mathrm{MIG}<\mathrm{TG} ; P<0.05)$ at the moment of traction of the first ovarian pedicle $(\mathrm{M} 2)$. Pain assessment by VAS showed statistical difference $24 \mathrm{~h}$ after the end of surgery $(\mathrm{TG}<\mathrm{MIG})(P<0.05)$.

Discussion: Both procedures were similar regarding intraoperative nociception and acute postoperative pain. It is possible that the sensation of pain in both procedures was blocked by the effectiveness of analgesics, once they might cause an inhibition of painful behaviors limiting a possible difference in pain identification. Higher respiratory stress observed in MIG at M2 and higher pain score by VAS noted in MIG $24 \mathrm{~h}$ after the end of surgery can be justified by greater traction of ovarian pedicle, due to limited surgical access of minimally invasive technique. Minimally invasive ovariohysterectomy non-laparoscopic seems to be potentially faster, probably due to the smaller size of the abdominal incision, which takes less time to be closed. In the present study, both techniques were performed by an experienced surgeon, providing safe procedures, nevertheless it is important to emphasize that iatrogenic injury can be caused by surgeons non-proficient in the Snook hook technique, considering the limited visualization of anatomical abdominal structures. Data obtained indicate that traditional ovariohysterectomy and non-laparoscopic Snook hook technique promote similar intraoperative nociception and acute postoperative pain, however minimally invasive procedure is potentially faster with less surgical trauma.
\end{abstract}

Keywords: canine, castration, ovary, Snook hook. 


\section{INTRODUCTION}

Ovariohysterectomy $(\mathrm{OH})$ is one of the most common surgical procedures performed in veterinary practice. The traditional surgical technique has been established and involves midline abdominal incision and removal of both ovaries and uterus. Later, minimally invasive techniques have been described $[23,26]$ and their benefits and potential complications have been widely discussed, especially with regard to postoperative pain [3,27].

Pain is a common $\mathrm{OH}$ postoperative complication, especially due an excessive manipulation and traction of tissues, and requires criterious attention [13]. The identification and prevention of pain and prolonged stress in patients undergoing surgical procedures are crucial for optimal recovery [15]. Several studies have demonstrated the advantages of using laparoscopy in comparison to traditional $\mathrm{OH}$, highlighting reduced intensity of pain, surgical stress and an early return to activities [13].

Considering the high cost, long learning curve and some limitations of laparoscopic $\mathrm{OH}$, the non-laparoscopic Snook hook technique can be an excellent alternative for performing minimally invasive $\mathrm{OH}$. This technique is straightforward and involves few equipment and a relatively shorter learning curve compared to laparoscopy, although meticulous training is mandatory [25].

The aim of this study was to evaluate surgical duration, intraoperative nociception and acute postoperative pain after traditional $\mathrm{OH}$ or minimally invasive non-laparoscopic mini-celiotomy by Snook hook technique in dogs. We hypothesized that non-laparoscopic minimally invasive $\mathrm{OH}$ would be faster and less painful than the conventional technique.

\section{MATERIALS AND METHODS}

\section{Animals}

Prior to selection, informed consent was obtained from all owners in the castration campaign. Thirty bitches, weighing 8 to $16 \mathrm{~kg}$ were used. The animals were received $24 \mathrm{~h}$ before the surgical procedure and evaluated for health (category ASA 1 - American Society of Anesthesiologists), through clinical and hematological examination. Exclusion criteria were dogs with abnormal laboratory values, cardiac arrhythmias, those in estrus or those with body condition scores greater than 6 on a 9-point scale. The patients were housed in individual cages where they received commercial feed and water ad libitum. Patients were randomly divided into 2 groups: Traditional Group $(\mathrm{TG}=15)$, submitted to conventional $\mathrm{OH}$ technique and Minimally Invasive Group (MIG = 15), submitted to Snook hook $\mathrm{OH}$ technique.

Surgical procedure

Patients were admitted to the veterinary teaching hospital at least $24 \mathrm{~h}$ prior to surgery for acclimatization. After $8 \mathrm{~h}$ fasting from solid food, bitches were premedicated with morphine [Dimorf ${ }^{\circledR 1}-5 \mathrm{mg} / \mathrm{kg}$ intramuscular (IM)]. Thirty min later, a $20 \mathrm{G}$ catheter $\left(\right.$ Insyte $\left.^{2}\right)$ was placed in the cephalic vein and anesthesia was induced with propofol IV [Diprivan ${ }^{\circledR 3}-5-7 \mathrm{mg}$ / $\mathrm{kg}$ ]. Maintenance was achieved with isoflurane [Forane $^{\circledR 4}$ diluted in $100 \%$ of oxygen]. Lactated Ringer's solution (Ringer Lactato ${ }^{5}$ ) was administered IV during anesthesia at a rate of $10 \mathrm{~mL} / \mathrm{kg} / \mathrm{h}$, immediately before the start of the surgery, single dose of fentanyl [Fentanil ${ }^{\circledR 6}-0.0025 \mathrm{mg} / \mathrm{kg}$ IV] was administered, diluted in $3 \mathrm{~mL}$ of saline solution [Sodium chloride solution ${ }^{\circledR 7}$ ], for $1 \mathrm{~min}$.

All ovariohysterectomy, traditional or minimally invasive, were performed by the same experienced surgeon. Bitches from TG were submitted to a conventional $\mathrm{OH}[12]$, using a median celiotomy of 5 $\mathrm{cm}$ of length retro-umbilical, ovaries and uterine bodies were exteriorized manually, following permanent hemostasis and removal. For animals from MIG, a mini-celiotomy of $1 \mathrm{~cm}$ of length was used, the suspensory ligament was ruptured, from where ovaries and uterine horns were identified and isolated with the aid of Snook hook [10]. In both groups, 3 Halsted hemostatic forceps were placed for ligation of ovarian pedicles using nylon suture (2-0) [Ethicon ${ }^{\mathrm{TM}}$ ]; then, the body of uterus was exposed, and 3 hemostatic forceps were applied immediately cranial to the cervix; bilateral ligation of uterine arteries and transfixation of the body of the uterus with nylon 0 were performed. The abdominal wall was sutured in a Sultan pattern with polyglactin $9100^{8}$, and the subcutaneous tissue in a simple continuous pattern with polyglactin $9102-0^{8}$. And, finally, the skin was sutured with 3-0 monofilament nylon ${ }^{8}$, in a simple interrupted pattern.

At the end of surgery, before extubation, 0.2 $\mathrm{mg} / \mathrm{kg}$ of meloxicam IM [Maxican ${ }^{\circledR 9}$ ] and $25 \mathrm{mg} / \mathrm{kg}$ of dipyrone IM [Novalgina injectable ${ }^{\circledR 10}$ ] were admin- 
istered. Curatives with gauze pads and microporous bandages of identical dimensions were applied to the surgical wounds to facilitate.

\section{Intraoperative assessment}

Heart rate (HR), respiratory rate $(f)$, systolic blood pressure (SBP) measured by the Doppler, esophageal body temperature (BT), oxyhemoglobin saturation ( $\mathrm{SpO} 2)$, end-tidal carbon dioxide concentration (EtCO2) and end-tidal isoflurane concentration were measured by multi-parameter monitor (Dixtal ${ }^{\mathrm{TM}}$ DX2010 ${ }^{11}$ ), 5 min before the start of surgery [before administration of fentanyl] (moment 0 - M0), during incision of linea alba (moment 1 - M1), during clamping of first ovarian pedicle (M2), during clamping of second ovarian pedicle (M3), during clamping of uterine cervix (M4), during suture of midline abdominal incision (M5), and at the end of the surgery (M6). The average duration of surgeries was also recorded, considering the moment of incision as the beginning of the surgery, until the last skin suture. The evaluation process at all times in this study was carried out blindly.

\section{Postoperative assessment}

In the postoperative period all animals were relocated in individual kennels. Evaluations were performed at 1, 2, 3, 4, 6, 8, and $24 \mathrm{~h}$ after the end of surgery.

For global assessment of pain intensity, the Glasgow Scale modified [22] was used, which is based on behavioral signs, and it is mainly used for assessment of acute postoperative pain. Concomitantly, for assessment of Global Pain Score, a Visual Analogue Scale (VAS) was applied, with a line from 0 to $100 \mathrm{~mm}$, where 0 indicates no pain and 100 the worst possible picture. This same scale was used to assess the surgical wound by palpation, where 0 indicates no reaction to palpation and 100 the worst possible reaction. The assessments were all made by one observer.

The degree of sedation was assessed at the same predetermined times, also by means of a visual analogue scale [line 0 to $100 \mathrm{~mm}$, where extreme left (0) indicates animal completely awake/alert and extreme right (100) unconscious animal].

Rescue analgesia was performed on animals with a score greater than or equal to 3 points on modified Glasgow Scale, associated with VAS of sedation equal to or less than $50 \mathrm{~mm}$. These animals received analgesia with application of morphine ${ }^{1}[0.5 \mathrm{mg} / \mathrm{kg}$,
IM]. After administration of rescue analgesia, evaluations were maintained at predetermined times.

At eight h postoperatively, tramadol [Tramadol Hydrochloride $^{12}$ - $4 \mathrm{mg} / \mathrm{kg} \mathrm{SC]}$ was administered for patients with no rescue analgesia. At the end of evaluation (24 h postoperatively), meloxicam ${ }^{9}[0.1 \mathrm{mg} / \mathrm{kg}$, $\mathrm{SC}]$ was reapplied in all animals.

\section{Statistical analysis}

The variables obtained during operation, such as $\mathrm{ET}_{\mathrm{ISO}}, \mathrm{EtCO}_{2}, \mathrm{HR}, f, \mathrm{SBP}, \mathrm{BT}$ and $\mathrm{SpO} 2$, were analyzed through Analysis of Variance (ANOVA) for 2 factors (group and time), and when significant differences were found, Tukey's test was used for multiple comparisons. Body weight, time of anesthesia and time of surgery were analyzed using the $t$ test for independent samples. Data from Glasgow Pain Scale, VAS of sedation, VAS of global assessment of pain intensity, VAS of palpation of wound, and age did not show normal distribution by the Kolmogorov-Smirnov test and were analyzed by the Mann-Whitney test, and in observing significant differences, Dunn's test was used for multiple comparisons. The results were presented as medians and 1 st and 3rd quartiles. The level of significance established for all statistical analysis mentioned above was 5\% $(P<0.05)$.

\section{RESULTS}

The assessment between groups regarding body weight $(\mathrm{TG}=12.5 \mathrm{~kg} ; \mathrm{MIG}=11 \mathrm{~kg} ; P=0.194)$, age $(\mathrm{TG}=2.1$ years; $\mathrm{MIG}=2.0$ years; $\mathrm{P}=0.667)$, duration of anesthesia (TG $=56 \mathrm{~min} ; \mathrm{MIG}=49 \mathrm{~min} ; P=$ $0.155)$ and time of surgery $(\mathrm{TG}=41 \mathrm{~min} ; \mathrm{MIG}=34$ $\min ; P=0.087$ ) did not show any significant difference.

The variables $\mathrm{ET}_{\text {ISO }}, \mathrm{HR}, \mathrm{BT}, \mathrm{SBP}$ and $\mathrm{SpO} 2$ measured peri and intraoperatively were not statistically different $(P>0.05)$ between moments and groups. However, EtCO2 (Table 1) and $f$ (Table 2) showed statistical difference in M2, being EtCO2 higher in TG and $f$ higher in MIG.

The results obtained from postoperative acute pain evaluation measured by Glasgow Scale, VAS of pain during palpation of surgical wound and VAS sedation did not present statistical difference between groups in any of the evaluated moments. However, VAS score of global pain (Table 3) showed statistical difference at $24 \mathrm{~h}$ after surgery, and MIG had higher pain score compared to TG. 
Supplementary analgesia in the first 8 postoperative $\mathrm{h}$ was performed for 6 animals from $\mathrm{TG}(48 \%)$ and six from MIG (48\%), with no difference between groups. In TG, 4 animals needed only one analgesic rescue ( 2 animals in the first h, 1 in the second and 1 in the third postoperative $\mathrm{h}$ ). Another animal required 3 analgesic rescues (1st, 2 nd, and 3 rd postoperative $h$ ); and a single animal required 4 analgesic rescues (1st, 4th, 6th, and 8th h postoperatively).

In MIG, 4 animals needed only 1 analgesic rescue ( 2 animals in the first postoperative $h$ and 2 animals in the second postoperative $h$ ); 2 other animals required 2 analgesic rescues ( 1 in the 1 st and 2 nd $h$ after surgery, and the other in the 2 nd and 6th $\mathrm{h}$ postoperatively).

Table 1. Mean and standard deviation of end-tidal carbon dioxide concentration $\left(\mathrm{ETCO}_{2}-\mathrm{mmHg}\right)$ in transoperative period of bitches submitted to ovariohysterectomy by traditional technique (TG) or minimally invasive (MIG) for postoperative pain assessment.

\begin{tabular}{|c|c|c|c|c|c|c|c|}
\hline \multirow{2}{*}{ Group } & \multicolumn{7}{|c|}{$\mathrm{ETCO}_{2}(\mathrm{mmHg})-$ Transoperative period } \\
\hline & M0 & M1 & M2 & M3 & M4 & M5 & M6 \\
\hline $\mathrm{TG}$ & $43 \pm 5$ & $37 \pm 13$ & $36 \pm 10^{\mathrm{a}}$ & $39 \pm 9$ & $42 \pm 7$ & $42 \pm 6$ & $42 \pm 3$ \\
\hline MIG & $39 \pm 7$ & $39 \pm 14$ & $28 \pm 16^{b}$ & $35 \pm 17$ & $48 \pm 9$ & $49 \pm 5$ & $46 \pm 5$ \\
\hline$P$-valor & 0.307 & 0.494 & 0.04 & 0.248 & 0.166 & 0.083 & 0.273 \\
\hline
\end{tabular}

$* P<0.05$ indicates a statistically significant difference. Different letters in columns indicate statistical difference between groups.

Table 2. Mean and standard deviation of respiratory rate $(f)$ (movements per minute - mpm) in transoperative period of bitches submitted to ovariohysterectomy by traditional technique (TG) or minimally invasive (MIG) for postoperative pain assessment.

\begin{tabular}{ccccccccc}
\hline \multirow{2}{*}{ Group } & \multicolumn{7}{c}{$f$ - Transoperative period } \\
\cline { 2 - 7 } & M0 & M1 & M2 & M3 & M4 & M5 & M6 \\
\hline TG & $16 \pm 8$ & $17 \pm 12$ & $21 \pm 12^{\mathrm{b}}$ & $21 \pm 14$ & $25 \pm 30$ & $15 \pm 6$ \\
MIG & $13 \pm 7$ & $17 \pm 17$ & $35 \pm 27^{\mathrm{a}}$ & $30 \pm 23$ & $18 \pm 16$ & $12 \pm 6$ & $14 \pm 6$ \\
$P$-valor & 0.605 & 0.927 & 0.014 & 0.091 & 0.223 & 0.369 & 0.809 \\
\hline
\end{tabular}

$* P<0.05$ indicates a statistically significant difference. Different letters in columns indicate statistical difference between groups.

Table 3. Medians ( $1^{\text {st }}$ and $3^{\text {rd }}$ quartiles) of pain scores measured by Visual Analog Scale (VAS) - global score - $(0$ - 100 points) in postoperative period of bitches submitted to ovariohysterectomy by traditional technique (TG) or minimally invasive (MIG).

\begin{tabular}{|c|c|c|c|c|c|c|c|}
\hline \multirow{2}{*}{ Group } & \multicolumn{7}{|c|}{ Postoperative period (hours) } \\
\hline & $1 \mathrm{~h}$ & $2 \mathrm{~h}$ & $3 \mathrm{~h}$ & $4 \mathrm{~h}$ & $6 \mathrm{~h}$ & $8 \mathrm{~h}$ & $24 \mathrm{~h}$ \\
\hline \multirow{2}{*}{ TG } & 31.5 & 19.5 & 14 & 14 & 3.5 & 3 & $0^{\mathrm{b}}$ \\
\hline & $(11.5-56.5)$ & $(9.0-37.0)$ & $(3.5-24.0)$ & $(3.5-25.0)$ & $(2.0-5.0)$ & $(0.0-9.0)$ & $(0.0-0.0)$ \\
\hline \multirow{2}{*}{ MIG } & 33 & 18.5 & 10 & 6 & 3.5 & 2 & $0,5^{\mathrm{a}}$ \\
\hline & $11.0-61.0)$ & $(4.0-50.0)$ & $(3.0-25.0)$ & $(3.0-23.0)$ & $(2.0-25.0)$ & $(0.0-3.0)$ & $(0.0-8.0)$ \\
\hline$P$-value & 0.918 & 0.918 & 0.816 & 0.378 & 0.835 & 0.600 & 0.026 \\
\hline
\end{tabular}

$* P<0.05$ indicates a statistically significant difference. Different letters in columns indicate statistical difference between groups.

\section{DISCUSSION}

An $\mathrm{OH}$ represents one of the most performed veterinary surgical procedures around the world and, contrary to popular belief, it is potentially risky and painful [1]. Several different variations of the technique have been described, however, it is known that minimally invasive approaches have a number of advantages, justifying their preference among more experienced surgeons [23]. Despite of published comparisons between conventional technique and minimally invasive laparoscopic $\mathrm{OH}$ [8], information about non-laparoscopic minimally invasive Snook hook $\mathrm{OH}(\mathrm{miOH})$ is lacking, especially with regard to intraoperative nociception and postoperative pain. 
Surprisingly, our results cannot accept the initial hypothesis that a minimally invasive technique would be less painful, but it was shown $12.5 \%$ to be faster and clearly less traumatic.

Although statistically significant differences between 2 techniques were not observed considering total surgical and anesthetic time, our results showed $17.1 \%$ and $12.5 \%$ faster for MIG, respectively. Especially for an experienced surgeon, identifying and exposing the ovaries and uterus is likely to take similar time between these 2 techniques, however it would take longer to incise and suture the surgical wound when performing the conventional one. Even though this numeric difference might be considered statically irrelevant, it is likely to be related to higher morbidity. A human study reported that the surgical infection rates can increase 5\% and $13 \%$ for every 10 and $15 \mathrm{~min}$ increase in surgical duration, respectively [7]. Additionally, the smaller size of the abdominal incision and area of cavity exposition can potentially decrease surgical trauma and time, reducing surgical infection $[17,19]$. These factors can also be responsible for shorter anesthetic time and drug consumption [28].

In the present study, miOH performed by an experienced surgeon (BWM) proved to be safe and easy to perform, although there may be more morbidity for surgeons non-proficient in the Snook hook technique [26], especially due to the fact that abdominal incision is smaller and, thus, limits the visualization of anatomical abdominal structures. In this scenario, surgical manipulation and trauma would be potentially higher [21]. Additionally, when organ adhesions to the visceral peritoneum are present, the use of the Snook hook through the surgical wound can cause iatrogenic injury [26].

The animals remained in surgical anesthetic plan throughout the procedure, presenting physiological parameters within the reference limits for the species. However, at moment of traction of the right ovary (M2), EtCO2 levels were higher in traditional $\mathrm{OH}$ group (TG) and respiratory rate $(f)$ was higher in MIG, because although the small incisions, there is still greater traction in ovarian pedicle for exposure and complete section, despite little manipulation of abdominal tissues and organs, which may have determined greater respiratory stress in MIG.

The ability to expose both ovaries and the body of the uterus through the same small celiotomy represents an additional limitation of $\mathrm{miOH}$. It is unlikely to achieve this objective without considering over pulling one of these structures, frequently the right ovary [10]. Ovarian pedicle clamping and ligation provided stimulation of severe pain. The manipulation of these receptors triggers nociception and demonstrates a stimulus-response relationship. Nociception is the mechanism of how this pain is felt, modulated and transmitted by the central nervous system; this mechanism is promoted by a highly specialized network of nociceptors and sensory neurons [18].

The animals that presented elevated $f$ were supplemented with greater vaporization of isoflurane to maintain the anesthetic plan, since this volatile agent triggers minimal cardiovascular effects and maintains cardiac output [24]. EtCO2, a variable referring to partial pressure of expired $\mathrm{CO} 2$, is one of the parameters that aimed to assess respiratory efficiency during the anesthetic procedure [20], and has an intimate relationship with $\mathrm{PaCO} 2$, which is insignificantly greater than previous and clinically irrelevant [29]. Therefore, under normal ventilation conditions, $\mathrm{PaCO} 2$ oscillates between 35 and $45 \mathrm{mmHg}$ [14], which corroborates the results obtained in this research, since the means found in TG and MIG groups were, respectively, $36 \pm 10 \mathrm{mmHg}$ and $28 \pm 16 \mathrm{mmHg}$, being within the physiological parameters. Except as previously discussed, the group MIG showed a hyperventilation condition that may be related to M2 pedicle traction pain.

It is known that $\mathrm{OH}$ causes moderate pain in the postoperative period and that its intensity depends on the duration and extent of the procedure, degree of tissue manipulation, age and body [6]. In this research, there was no statistical difference in relation to the VAS of wound palpation, sedation and pain score using the Glasgow Scale. Standardized anesthetic protocol and an experienced surgeon were chosen in order to mitigate a potential bias on these assessments [4]. However, it is possible that the sensation of pain in both procedures was blocked by the effectiveness of analgesics, once they might cause an inhibition of painful behaviors limiting a possible difference in pain identification.

The assessment of global pain score by VAS resulted in a statistical difference $24 \mathrm{~h}$ after 
the end of surgery. At that time, 1 animal from TG and 7 from the MIG had pain scores, which were 3 points for the first group mentioned and 1 , $2,3,8,11,14$, and 26 points for the second. Data that do not corroborate Jacobsen et al. [16], who describe that minimally invasive techniques in horses cause less inflammation and postoperative pain when compared to corresponding conventional surgical techniques. Souza et al. [26] compared conventional, minimally invasive and laparoscopic $\mathrm{OH}$, and observed no difference in postoperative recovery of animals. However, the results of present study can be justified by greater traction of ovarian pedicle, due to limited surgical access of minimally invasive technique, which can result in a higher level of nociception [10].

In Glasgow Scale, this score is $\geq 6$ and in VAS is $50 \mathrm{~mm}$ [9]. In this study, a score $\geq 3$ for Glasgow Scale was used, as it was described that some animals with scores between 38 and $48 \%$ of total presented clinical signs of discomfort, but did not benefit from the use of rescue analgesia [2]. This data is important, as it prevents unnecessary suffering towards patients (samples) during the execution of research and, additionally, prevents inaccurate conclusions from being promoted by researchers [5].

The main limitation of this study refers to the lack of analysis of glucose and serum cortisol, which are useful in magnifying surgical stress and hospitalization [11]. However, we believe that our results allow us to adequately embrace the proposed objectives.

\section{CONCLUSION}

Both Ovariohysterectomy $(\mathrm{OH})$ techniques were found to be similar regarding intraoperative nociception and acute postoperative pain. Minimally invasive non-laparoscopic $\mathrm{OH}$ is potentially faster with less surgical trauma.

\section{MANUFACTURERS}

${ }^{1}$ Cristália Produtos Químicos Farmacêuticos Ltda. Itapira, SP, Brazil. ${ }^{2}$ Becton Dickinson Indústrias Cirúrgicas Ltda. Curitiba, PR, Brazil.

${ }^{3}$ AstraZeneca do Brasil Ltda. São Paulo, SP, Brazil.

${ }^{4}$ Abbott Laboratorios do Brasil Ltda. Rio de Janeiro, RJ, Brazil.

${ }^{5} J P$ Indústria Farmacêutica S.A. Ribeirão Preto, SP, Brazil.

${ }^{6} J a n s s e n-C i l a g$ Farmacêutica Ltda. São José dos Campos, SP, Brazil. ${ }^{7}$ Isofarma Industrial Farmacêutica Ltda. Eusébio, CE, Brazil.

${ }^{8}$ Johnson \& Johnson do Brasil Indústria e Comércio de Produtos para Saúde Ltda. São José dos Campos, SP, Brazil.

${ }^{9}$ Ouro Fino Saúde Animal Ltda. Cravinhos, SP, Brazil.

${ }^{10}$ Sanofi-Aventis Farmacêutica Ltda. São Paulo, SP, Brazil.

${ }^{11}$ Dixtal Biomédica Indústria e Comércio Ltda. São Paulo, SP, Brazil.

${ }^{12}$ Medley Farmacêutica Ltda. Campinas, SP, Brazil.

Funding. This project was supported by the São Paulo Research Foundation (FAPESP - number 2013/10871-9).

Acknowledgments. The authors thank Juliana Tabarelli Brondani and André Escobar for anaesthetic support, Letícia Camargo Todaro Tucci for reseach support, and FAPESP (2013/10871-9) for the scholarship granted.

Ethical approval. This study was approved by the Committee on Ethics in the Use of Animals (CEUA), Universidade Estadual Paulista (Unesp), campus Jaboticabal (Protocol number 007279/13).

Declaration of interest. The authors report no conflicts of interest. The authors alone are responsible for the content of the paper.

\section{REFERENCES}

1 Adin C.A. 2011. Complications of ovariohysterectomy and orchiectomy in companion animals. Veterinary Clinics: Small Animal Practice. 41(5): 1023-1039.

2 Al-gizawiy M.M. \& Rudé E.P. 2004. Comparison of preoperative carprofen and postoperative butorphanol as postsurgical analgesics in cats undergoing ovariohysterectomy. Veterinary Anaesthesia and Analgesia. 31(3): 164-174.

3 Burrow R., Batchelor D. \& Cripps P. 2005. Complications observed during and after ovariohysterectomy of 142 bitches at a veterinary teaching hospital. Veterinary Record. 157(26): 829-833.

4 Brondani J.T., Luna S.P.L. \& Padovani C.R. 2011. Refinement and initial validation of a multidimensional composite scale for use in assessing acute postoperative pain in cats. American Journal of Veterinary Research. 72(2): 174-183.

5 Brondani J.T., Luna S.P.L., Minto B.W., Santos B.P.R., Beier S.L., Matsubara L.M. \& Padovani C.R. 2013. Reliability and cut-off point related to the analgesic intervention of a multidimensional composite scale to assess postoperative pain in cats. Brazilian Journal of Veterinary and Animal Sciences. 65(1): 153-162. 
6 Campagnol D., Teixeira Neto F.J., Monteiro E.R., Restitutti F. \& Minto B.W. 2012. Effect of intraperitoneal or incisional bupivacaine on pain and the analgesic requirement after ovariohysterectomy in dogs. Veterinary Anaesthesia and Analgesia. 39(4): 426-430.

7 Cheng H., Chen B.P.H., Soleas I.M., Ferko N.C., Cameron C.G. \& Hinoul P. 2017. Prolonged operative duration increases risk of surgical site infections: a systematic review. Surgical infections. 18(6): 722-735.

8 Corriveau K.M., Giuffrida M.A., Mayhew P.D. \& Runge J.J. 2017. Outcome of laparoscopic ovariectomy and laparoscopic-assisted ovariohysterectomy in dogs: 278 cases (2003-2013). Journal of the American Veterinary Medical Association. 251(4): 443-450.

9 Coutinho A.F.O.S.V. 2012. Subjectivity on animal pain assessment. 79f. Lisboa, Portugal. Dissertation (Integrated Master in Veterinary Medicine) - Faculty of Veterinary Medicine, Technical University of Lisbon.

10 Coutinho A.J., Gasser B., Rodriguez M.G.K., Uscategui R.A.R., Santos V.J.C., Tiosso C.D.F., Barros F.F.P.C \& Toniollo G.H. 2018. Comparison between single port videolaparoscopy and miniceliotomy with snock hook ovariohysterectomy techniques in bitches. Ciência Rural. 48(10): 1-9.

11 Devitt C.M., Cox R.E. \& Hailey J.J. 2005. Duration, complications, stress, and pain of open ovariohysterectomy versus a simple method of laparoscopic-assisted ovariohysterectomy in dogs. Journal of the American Veterinary Medical Association. 227(6): 921-927.

12 Fransson B.A. 2018. Ovaries and Uterus. In: Johnston S.A. \& Tobias K.M. (Eds). Veterinary Surgery Small Animal. 2nd edn. St. Louis: Elsevier, pp.4893-4896.

13 Hancock R.B., Lanz O.I., Waldron D.R., Duncan R.B., Broadstone R.V. \& Hendrix P.K. 2005. Comparison of postoperative pain after ovariohysterectomy by harmonic scalpel-assisted laparoscopy compared with median celiotomy and ligation in dogs. Veterinary Surgery. 34(3): 273-282.

14 Haskins S.C. 1996. Monitoring the anesthetized patient. In: Thurmon J.C., Tranqulli W.J. \& Benson G.J. (Eds). Lumb \& Jones'veterinary anesthesia. 3rd edn. Baltimore: Lea \& Febiger Book, pp.409-424.

15 Hellyer P., Rodan I., Brunt J., Downing R., Hagedorn J.E. \& Robertson S.A. 2007. AAHA/AAFP pain management guidelines for dogs and cats. Journal of Feline Medicine \& Surgery. 9(6): 466-480.

16 Jacobsen S., Jensen J.C., Frei S., Jensen A.L. \& Thoefner M.B. 2005. Use of serum amyloid A and other acute phase reactants to monitor the inflammatory response after castration in horses: a field study. Equine Veterinary Journal. 37(6): 552-556.

17 Khalaj A., Bakhtiari J. \& Niasari-Naslajl A. 2012. Comparison between single and three portal laparoscopic splenectomy in dogs. BMC Veterinary Research. 8(161): 1-4.

18 Lamont L.A., Tranquilli W.J. \& Grimm K.A. 2000. Physiology of pain. Veterinary Clinics of North America: Small Animal Practice. 30(4): 703-728.

19 Mayhew P.D., Freeman L., Kwant T. \& Brown D.C. 2012. Comparison of surgical site infection rates in clean and clean-contaminated wounds in dogs and cats after minimally invasive versus open surgery: 179 cases (2007-2008). Journal of the American Veterinary Medical Association. 240(2): 193-198.

20 Moon R.E. 1990. Respiratory monitoring. In: Miller R.D. (Ed). Anesthesia. 4th edn. New York: Churchill Livingstone, pp.1129-1163.

21 Mifilari R. \& Vuono R.S. 2000. Ováriosalpingohisterectomia em cadelas e gatas: proposta de novos procedimentos. Revista de Educação Continuada CRMV-SP. 3(3): 28-32.

22 Murrel J.C., Psatha E.P., Scott E.M., Reid J. \& Hellebrekers L.J. 2008. Application of a modified form of the Glasgow Pain Scale in a veterinary teaching center in the Netherlands. Veterinary Record. 162(13): 403-408.

23 Peeters M.E. \& Kirpensteijn J. 2011. Comparison of surgical variables and short-term postoperative complications in healthy dogs undergoing ovariohysterectomy or ovariectomy. Journal of American Veterinary Medical Association. 238(2): 189-194.

24 Preckel B., Mullenhem J., Hoff J., Obal D., Heiderhoff M., Thamer V. \& Schlack W. 2004. Haemodynamic changes during halothane, sevoflurane and desflurane anaesthesia in dogs before and after the induction of severe heart failure. European Journal Anaesthology. 21(10): 797-806.

25 Quessada A.M., Sousa A.A.R., Costa A.P.R., Sousa A.A.S. \& Rocha R.R.C. 2009. Comparison among ovariohysterectomy techniques in bitches. Acta Scientiae Veterinariae. 37(3): 253-258. 
26 Souza F.W., Brun M.V., Oliveira M.T., Feranti J.P.S., Corrêa R.K.R., Idalêncio R., Duda N.C.B., Quadros A.M. \& Huppes R.R. 2014. Ovariohysterectomy for videosurgery (hybrid vaginal NOTES), celiotomy or mini-celiotomy in bitches. Ciência Rural. 44(3): 510-516.

27 Spain C.V., Scarlett J.M. \& Houpt K.A. 2004. Long-term risks and benefits of early-age gonadectomy in dogs. Journal of the American Veterinary Medical Association. 224(3): 380-387.

28 Steagali P.V.M., Taylor P.M., Rodrigues L.C.C., Ferreira T.H., Minto B.W. \& Aguiar A.J. 2009. Analgesia for cats after ovariohysterectomy with either buprenorphine or carprofen alone or in combination. Veterinary Record. 164(12): 359-363.

29 Teixeira Neto F.J., Carregaro A.B., Mannarino R., Cruz M.L. \& Luna S.P. 2002. Comparison of sidestream capnograph and a mainstream capnograph in mechanically ventilated dogs. Journal of the American Veterinary Medical Association. 221(11): 1582-1585. 